\title{
Datenbasis und Sampling
}

\subsection{Datensätze zur Bestimmung der Bruttostichprobe der MSO}

$\mathrm{Zu}$ Beginn der Durchführung der quantitativen Studie stand die Autorin dieser Arbeit vor der Herausforderung, sich ein Bild über den Forschungsgegenstand in der Breite machen zu müssen und war entsprechend mit der Frage konfrontiert, wie sich anhand festgelegter Definitionskriterien Bruttostichproben für Deutschland, einzelne Bundesländer oder Kommunen bestimmen lassen.

Aufgrund der für diese Arbeit geltend gemachten weiten Definition von MSO - verstanden als von einst zugewanderten Menschen oder deren Nachkommen gegründeten Organisationen, deren Mitglieder mehrheitlich Menschen mit einer Zuwanderungsgeschichte sind - wurde von einer maximalen Anzahl von für die Grundgesamtheit gültigen Organisationen ausgegangen. Bei Zugrundelegung einer enger gefassten (normativen) Definition, etwa nach Generationenzugehörigkeiten der Mitglieder oder integrationspolitischen Ausrichtungen, hätten die recherchierten MSO ohnehin vor einer Befragung nicht gesondert nach spezifischen Eigenschaften sortiert bzw. selektiert werden können.

Eine schriftliche Befragung von MSO setzt grundlegende, zeitaufwendige Vorarbeiten voraus. Profitieren konnte die Autorin dieser Studie von der Arbeit des Stifterverbandes Berlin für den ZiviZ-Survey 2017 (ZiviZ: „Zivilgesellschaft in Zahlen“; Priemer et al. 2017): Auf Basis des Vereinsregisters $2016^{1}$ wurde eine

\footnotetext{
${ }^{1}$ Datenbasis: Vereinsregister 2016; gemeinsames Registerportal der Länder, Suchabfragen unter: https://www.handelsregister.de (Priemer et al. 2017: 41, 50).
} 
Gesamtliste aller Vereine in Deutschland generiert und vermittels einer Namensanalyse nach bestimmten Begrifflichkeiten ${ }^{2}$ eine Gesamtzahl von 17.500 MSO herausgefiltert (Priemer 2017; s. dazu auch I. 2.1). Diese Zahl korrespondiert mit anderen Zahlen, die für in Deutschland insgesamt ansässigen MSO ermittelt wurden (IntB 2011: 6). Die für NRW erzeugte Liste der MSO-Namen wurde der Autorin zur Verfügung gestellt, die diese wiederum nach den für die Vollerhebung ${ }^{3}$ ausgewählten Ruhrgebietsstädten und Bielefeld (5.2) gefiltert hat. Die Kontaktdaten der MSO mussten komplett selbst ausfindig gemacht werden. Da mehrere Wege der Kontaktaufnahme möglich sein sollten, wurden Post- und E-Mail-Adressen sowie Telefonnummern und nach Möglichkeit Namen von konkreten Ansprechpartnern, wie beispielsweise Vereinsvorsitzenden, recherchiert. Einige der in der Gesamtliste registrierten MSO waren gar nicht ausfindig zu machen und bei anderen stellte sich heraus, dass es sich nicht um migrantische Vereinigungen handelte.

Neben den ZiviZ-Daten konnte darüber hinaus auf die Kontaktdatenliste von religiösen Gemeinden in NRW zurückgegriffen werden, die im Rahmen der Studie „Religiöse Vielfalt in Nordrhein-Westfalen“ (Hero et al. 2008) erstellt und die im Jahr 2017 aktualisiert wurde. Auch aus dieser Liste wurden die migrantischen religiösen Gemeinden für die Erhebungskommunen herausgefiltert und mit den in der Liste des Stifterverbandes vorhandenen MSO abgeglichen. Ein großer Vorteil war, dass die Kontaktdaten hier bereits enthalten und auf den aktuellsten Stand gebracht worden waren. Darüber hinaus konnte die Autorin von Kontaktlisten profitieren, die ihr seitens Kommunaler Integrationszentren zur Verfügung gestellt wurden; aus datenschutzrechtlichen Gründen mussten darin enthaltene personenbezogene Daten vor der Übermittlung der Dateien jedoch gelöscht werden. Des Weiteren wurden MSO mithilfe des Internets recherchiert. Dabei stellte sich heraus, dass im Internet auffindbare Listen zum Teil sehr veraltete Informationen enthielten und nicht mehr aktuell waren. Auf Basis aller zu ermittelnden Kontaktdaten wurden integrierte Listen im Serienbrief-Format erstellt.

Insgesamt war die Recherche der Bruttostichprobe somit sehr explorativ angelegt. Es zeigten sich recht starke Abweichungen zwischen den im Vereinsregister

\footnotetext{
${ }^{2}$ Ein Verein wurde dann als MSO kategorisiert, wenn im Namen ein Raumbezug (z. B. „türkisch“) oder ein sich nicht auf die Mehrheitsgesellschaft beziehender Religionsbezug (z. B. ,islamisch“) erkennbar war. Darüber hinaus wurden bei der Filterung fremdsprachige Namen und weitere Begrifflichkeiten, die auf einen Migrationsbezug verweisen könnten (wie z. B. „Migration“ oder ,internationale Solidarität“), berücksichtigt (vgl. I. 2.1, Fußnote 3).

${ }^{3}$ Allein aufgrund der defizitären Datenlage ist das Ziehen einer repräsentativen Zufallsstichprobe nicht möglich. Insofern zeigt sich bei dieser Vorgehensweise der Charakter einer Pionier- bzw. Pilotstudie.
} 
eingetragenen Organisationen, den auf kommunaler Ebene verfügbaren Kontaktlisten sowie Informationen auf Internetseiten, die von Zusammenschlüssen bzw. Dachverbänden der MSO oder seitens der Städte erstellt wurden.

Die über mehrere Quellen recherchierbare Anzahl von MSO fiel in einigen Städten höher aus als die auf Basis der Liste des Stifterverbands Berlin sowie des Forschungsprojekts „Religiöse Vielfalt in Nordrhein-Westfalen“ festzustellenden Gesamtzahl (Tab. 5.1). Die recherchierte Gesamtzahl stellte die Grundlage für den Versand des Anschreibens und des Fragebogens dar.

Tab 5.1 Diskrepanzen zwischen ermittelten MSO-Zahlen (in absoluten Häufigkeiten)

\begin{tabular}{lcc}
\hline Erhebungskommunen & $\begin{array}{c}\text { Vereinsregister 2016 \& } \\
\text { NRW-Plural 2017 }\end{array}$ & Versand Anschreiben* \\
\hline Dortmund & 290 & 239 \\
\hline Essen & 189 & 185 \\
\hline Duisburg & 145 & 127 \\
\hline Bochum & 150 & 161 \\
\hline Gelsenkirchen & 123 & 111 \\
\hline Bielefeld & 113 & 177 \\
\hline Oberhausen & 43 & 79 \\
\hline Hagen & 75 & 66 \\
\hline Hamm & 61 & 99 \\
\hline Summe & 1.189 & $\mathbf{1 2 4 4}$ \\
\hline
\end{tabular}

* Insgesamt recherchierbare Adressen. Basis: deutsches Vereinsregister 2016; NRW-Plural 2017 (Hero et al. 2008 aktualisiert); Internetseiten; kommunale MSO-Listen.

\subsection{Auswahl der nordrhein-westfälischen Kommunen für die Erhebung}

Grundsätzlich ist immer auch von einer kontextabhängigen Ausprägung der zivilgesellschaftlichen Aktivitäten von MSO auszugehen (II. 3.2). Daher sind bei der Auswahl von Kommunen, in denen eine Befragung von MSO durchgeführt werden soll, Einflussfaktoren in den Blick zu nehmen, die sich potenziell auf die gesellschaftliche Beteiligung von Individuen und Kollektivakteuren auswirken. Diese Faktoren betreffen strukturelle Daten und die konkrete Ausgestaltung der Kommunalpolitik, die auch die Existenz von Koordinatorenstellen, Netzwerken und Kooperationen sowie historisch bedingte Erfahrungsgrößen einschließt (II. 3.2). Die benannten Größen manifestieren und kombinieren sich auf kommunaler Ebene unterschiedlich und können entsprechend ungleiche Wirkungen 
und Relevanzen für die zivilgesellschaftlichen Performanzen der MSO haben (BMFSFJ 2017: 352 ff.; Hameister/Tesch-Römer 2016; Klie 2019).

Kenngrößen oder „Strukturmerkmale“ von Regionen und Städten, wie die Einkommenssituation und Armutsgefährdung, die Wirtschaftsstruktur und Beschäftigung $^{4}$, sind grundsätzlich bedeutsam für die Bereitschaft, sich zu engagieren und bei der Ausprägung lokalspezifischer Rahmenbedingungen und Engagementniveaus als Erklärungsfaktoren heranzuziehen. In der Regel gilt: Je niedriger die Arbeitslosenquote ist, desto höher fällt der Anteil von freiwillig Engagierten aus (Hameister/Tesch-Römer 2016: 539 ff.). Bei der Auswahl der Kommunen, in denen die Erhebung durchgeführt wurde, war es ein nicht einfach zu realisierendes Anliegen, sich an der Existenz möglichst ähnlicher Strukturbedingungen zu orientieren. Die Strukturdaten der Kommunen sollten weitgehend gleich ausgeprägt sein, um differente Strukturmerkmale als stark intervenierende Variablen kontrollieren und Kontexteffekte weitestgehend ausschließen zu können (,Most-Similar“-Ansatz ${ }^{5}$ ). Als relevant erscheinen zuvorderst die Arbeitslosenquote und die Einkommenssituation (Primäreinkommen und verfügbares Einkommen der privaten Haushalte), die wiederum als Indikatoren für wirtschaftliche Stärke, Beschäftigung und Armutsgefährdung gelten können. ${ }^{6}$ Ausgewählt wurden daher zunächst sechs kreisfreie kleine und große Großstädte des Ruhrgebiets mit Arbeitslosenquoten zwischen 10 bis 13 Prozent (durchschnittliche Arbeitslosenquote in NRW: 8,5 \%): Dortmund, Essen, Bochum, Oberhausen, Hagen, Hamm. Mit Bielefeld wurde der Auslese eine kleine kreisfreie Großstadt

\footnotetext{
${ }^{4}$ Weitere für die Entfaltung und Leistungsfähigkeit von Engagement bedeutsame Ressourcen und Bedingungen wie die Dynamik der demografischen Entwicklung, die Wahlbeteiligung und politische Partizipation sowie die Ausstattung mit engagementfördernder Infrastruktur (BMFSFJ 2017: 352 ff.) wurden als kommunale Kennziffern vernachlässigt.

${ }^{5}$ Diese Vorgehensweise ist nicht zu verwechseln mit dem in der vergleichenden Politikwissenschaft für quantitative Studien auf makrostruktureller Ebene gängigen kontrollierten Forschungsdesign des Most Similar Cases Design (mscd). Dieses umfasst sehr ähnliche Fälle, in denen die ,abhängige Variable“ - ein Phänomen (Ergebnis) - in sehr ähnlichen Kontexten variiert: existent ist oder nicht. Die Faktoren, die als ursächlich für das Phänomen betrachtet werden (die „unabhängigen Variablen“) sind ähnlich, aber nicht identisch, sondern variieren kontrolliert, da mit ihnen ansonsten keine Unterschiede erklärt werden können. Das unterschiedliche Ergebnis (,y“, , nicht y“) ist mit der Differenz bei den verursachenden Faktoren zu erklären (,x2‘ und ,nicht x2‘) (Lauth et al. 2015: 62 f.).

${ }^{6}$ Im Rahmen der Analysen des Freiwilligensurveys wird ausschließlich die Arbeitslosenquote als Indikator für die wirtschaftliche Prosperität von Landkreisen und kreisfreien Städten herangezogen (Hameister/Tesch-Römer 2016: 541).
} 
in Ostwestfalen-Lippe hinzugefügt. Den ersten Recherchen zufolge sollten in diesen Städten insgesamt 1.006 MSO erreicht und befragt werden können (mit den größten Anteilen in Dortmund und Essen ${ }^{7}$ ).

Neben den strukturellen Merkmalen der Kommunen sollte zudem der Anteil der Menschen mit Migrationshintergrund (inklusive Ausländer) und deren Zusammensetzung nach Herkunftsländern berücksichtigt werden. In den drei Städten Essen, Oberhausen und Bochum lag der Anteil der Menschen mit Migrationshintergrund im Jahr 2016 zwischen 25 und 27 Prozent, in den anderen Städten bei über 30 Prozent (bei einem Durchschnitt von 25,8 \% in NRW) (Tab. 5.2). Die Unterschiede sind in den Ruhrgebietsstädten, als Schmelztiegel diverser Kulturkreise, hinzunehmen und wären auch mit einer Auswahl von anderen Städten, nicht zu nivellieren gewesen, da sich diese dann wiederum hinsichtlich anderer der hier primär berücksichtigten strukturellen Kriterien unterschieden hätten. Zudem haben in allen Kommunen die meisten MSO einen türkischen (bzw. islamischen) Hintergrund.

Es zeigte sich jedoch, dass viele der MSO in den sieben Städten auf dem Postweg nicht zu erreichen waren (5.3). Um das Sample der MSO und damit auch den möglichen Rücklauf zu erhöhen, wurden daher mit den Städten Duisburg und Gelsenkirchen zwei weitere Großstädte im Ruhrgebiet in das Sample aufgenommen. Den Recherchen zufolge beherbergen beide Städte jeweils über hundert MSO, darunter auch viele der etablierten islamischen Verbände DİTIB, VIKZ und IGMG. Duisburg und Gelsenkirchen, im Ballungsraum des Ruhrgebietes, weisen einen sehr hohen Bevölkerungsanteil von türkeistämmigen Menschen und den höchsten Anteil von Mitgliedern in bzw. Zugehörigen zu Moscheegemeinden in Nordrhein-Westfalen auf (Duisburg: $>7,0 \%$; Gelsenkirchen: 6 bis $>7 \%{ }^{8}$ ). Es handelt sich bei den beiden Städten jedoch um strukturschwächere Kommunen, die mit größeren sozialen und Integrationsherausforderungen konfrontiert sind. ${ }^{9}$

Mit der Hinzunahme von Duisburg und Gelsenkirchen als zwei strukturschwache Städte Nordrhein-Westfalens wurde damit vom in der Auswahl der

\footnotetext{
${ }^{7}$ Ohne die beiden Städte Dortmund und Essen hätten lediglich circa 582 MSO kontaktiert werden können.

${ }^{8}$ Die Berechnung des Anteils erfolgte in Relation zur Wohnbevölkerung der beiden kreisfreien Städte. Die Zahlen basieren über formale Mitgliedschaften hinausgehend auf Berechnungen der tatsächlich einer Gemeinde zugehörigen Personen (Hero et al. 2008: 229). Die Varianz liegt in NRW-Kommunen bei $<1,0$ bis $>$ 7,0 Prozent.

${ }^{9}$ Bestimmte Stadtteile wie Hochfeld und Marxloh in Duisburg oder im Gelsenkirchener Süden werden aufgrund von sozialen und ethnischen Polarisierungs- und Segregationstendenzen häufig als soziale Brennpunkte oder „Problemviertel“ etikettiert (Ceylan 2006; Cöster 2016). In diesem Zusammenhang relevant ist auch das von medialer Aufmerksamkeit begleitete Phänomen der südosteuropäischen „Armutszuwanderung“ durch die sozial schwachen Gruppen
} 
Kommunen zunächst verfolgten „Most-Similar-Ansatz“ deutlich abgewichen. Hintergrund der Entscheidung war auch, dass die Autorin im Rahmen der Umfragevorbereitungen ganz zu Beginn der Studie explorative Feldsondierungen und Experteninterviews mit kommunalpolitischen Akteuren, Fachpraktikern sowie Vertretern von MSO durchgeführt hat, die Anlass zur Vermutung gaben, dass die Städte und die dort ansässigen MSO trotz strukturpolitisch ungünstiger Umstände und Voraussetzungen als zivilgesellschaftlich aktiv und interessiert einzuschätzen sind. In diesem Zusammenhang sollte bedacht werden, dass Engagementprofile durch Strukturbedingungen keinesfalls prädeterminiert sind und ein für alle Mal feststehen (II. 3.2). Wenngleich sich Wohlstand positiv und eine hohe Arbeitslosigkeit negativ auf Engagementbeteiligung auswirken und Strukturbedingungen von Kommunen mit höheren Arbeitslosenquoten, geringeren durchschnittlichen Einkommen und einer schlechteren sozioökonomischen Lage der Bevölkerung das Engagementpotenzial in der Stadtgesellschaft unterminieren, sind auch andere Szenarien denkbar. Denn umgekehrt könnten gerade der Bedarf und die Notwendigkeit zivilgesellschaftlichen Engagements in Städten mit strukturellen Defiziten, die mit kumulierenden Problemlagen konfrontiert sind, zu vitalen zivilgesellschaftlichen Strukturen führen, die unter Umständen höher ausfallen können als in Kommunen mit einer geringeren Arbeitslosenquote, einer soliden finanziellen Ausstattung und größeren Handlungsspielräumen (Hameister/Tesch-Römer 2016: 541).

Bei der Auswahl der Kommunen ist die Beachtung der genannten Kontextfaktoren wichtig; gleichwohl ist einzuräumen, dass für die Feststellung von Ausmaß und Qualität der jeweiligen politischen, strukturellen, häufig auch durch Einzelpersonen geprägten Randbedingungen, komplexe (auch qualitative) Analysen der kommunalen Gegebenheiten erforderlich wären, die im Rahmen dieser Arbeit nicht geleistet werden konnten. Unabhängig davon ist an dieser Stelle jedoch zugleich zu konstatieren, dass selbst bei Kenntnis dieser Faktoren, die die Förderung, Einbindung und Aktivierung der MSO in unterschiedlichen Intensitätsgraden betreffen (II. 3.2), das Ziehen von Rückschlüssen im Sinne negativer oder positiver Einflusseffekte auf die zivilgesellschaftliche Performanz einzelner MSO bzw. die Veranschlagung von sich in den Daten mutmaßlich spiegelnden Effekten der Rahmenbedingungen ein hochspekulatives Vorgehen wäre.

Auch angesichts der mit dem ,Teilhabe- und Integrationsgesetz“ (2012) in NRW gesetzlich verankerten aktivierenden Integrationspolitik, einschließlich der

der Rumänen (z. T. Roma) und Bulgaren, die häufig eine ethnisch-kulturelle Diskriminierung durch ehemals Eingewanderte und Alteingesessene erfahren (Nurec-Institute Duisburg 2016a, b; Böckler et al. 2018; MKFFI NRW 2017: 4, 17). 
Tab.5.2 Profile der ausgewählten Kommunen (2016)

\begin{tabular}{|c|c|c|c|c|c|c|}
\hline \multirow[t]{2}{*}{ Stadt } & \multirow[t]{2}{*}{ Einwohner* } & \multirow[t]{2}{*}{$\begin{array}{c}\text { Menschen } \\
\text { mit } \text { MH*I }^{*} \\
\text { Ausländer } \\
\text { (NRW insg.: } \\
25,8 \% \text { ) }\end{array}$} & \multirow[t]{2}{*}{$\begin{array}{l}\text { Zuwanderungs- } \\
\text { gruppen* }\end{array}$} & \multirow[t]{2}{*}{$\begin{array}{l}\text { Arbeits- } \\
\text { losenquote } \\
\text { in \%* } \\
(\mathrm{NRW}: \\
8,5 \%)\end{array}$} & \multirow[t]{2}{*}{$\begin{array}{c}\text { Anzahl der } \\
\text { MSO* }^{*}\end{array}$} & \multirow{2}{*}{ 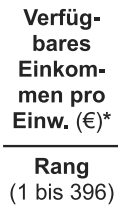 } \\
\hline & & & & & & \\
\hline \multirow[t]{4}{*}{ Dortmund } & \multirow[t]{4}{*}{585.813} & \multirow{4}{*}{$\begin{array}{l}32,1 \% \\
16,4 \%\end{array}$} & Türkei: $20,4 \%$ & \multirow[t]{4}{*}{13,0} & \multirow{4}{*}{$\begin{array}{r}290 \\
(\sim 5,1 \% \\
\text { der Vereine } \\
\text { in DO })\end{array}$} & 18.946 \\
\hline & & & $\begin{aligned} \text { Polen: } & 8,6 \% \\
\text { Syrien/Arab. Rep.: } & 7,8 \%\end{aligned}$ & & & 367 \\
\hline & & & Rumänien: $4,4 \%$ & & & \\
\hline & & & Griechenland: $3,8 \%$ & & & \\
\hline \multirow[t]{5}{*}{ Essen } & \multirow[t]{5}{*}{583.084} & \multirow{5}{*}{$\begin{array}{l}\mathbf{2 6 , 9} \% \\
14,7 \%\end{array}$} & Türkei: $16,3 \%$ & \multirow[t]{5}{*}{13,0} & \multirow{5}{*}{$\begin{array}{r}189 \\
(\sim 4,6 \% \\
\text { der Vereine } \\
\text { in } \mathrm{E})\end{array}$} & 20.159 \\
\hline & & & Syrien/Arab. Rep.: $9,9 \%$ & & & \\
\hline & & & Polen: $7,7 \%$ & & & 327 \\
\hline & & & Irak: $5,5 \%$ & & & \\
\hline & & & Serbien o. Kosovo: $3,9 \%$ & & & \\
\hline \multirow[t]{5}{*}{ Bochum } & \multirow[t]{5}{*}{364.920} & \multirow{5}{*}{$\begin{array}{l}25,6 \% \\
11,7 \%\end{array}$} & \multirow{5}{*}{$\begin{array}{rr}\text { Türkei: } & 18,9 \% \\
\text { Syrien/Arab. Rep.: } & 12,6 \% \\
\text { Polen: } & 7,9 \% \\
\text { Italien: } & 3,9 \% \\
\text { Rumänien: } & 3,6 \%\end{array}$} & \multirow[t]{5}{*}{11,1} & \multirow{5}{*}{$\begin{array}{r}150 \\
(\sim 3,5 \% \\
\text { der Vereine } \\
\text { in } \mathrm{BO})\end{array}$} & 19.620 \\
\hline & & & & & & \\
\hline & & & & & & 349 \\
\hline & & & & & & \\
\hline & & & & & & \\
\hline \multirow[t]{6}{*}{ Bielefeld } & 333.451 & $35,5 \%$ & Türkei: $20,2 \%$ & 9,7 & 114 & 22.659 \\
\hline & & $13,7 \%$ & Irak: $9,3 \%$ & & $(\sim 5 \%$ & \\
\hline & & & Griechenland: $\quad 6,6 \%$ & & der Vereine & 154 \\
\hline & & & Polen: $6,5 \%$ & & in $\mathrm{BI}$ ) & \\
\hline & & & Syrien/Arab. Rep.: $\quad 5,6 \%$ & & & \\
\hline & & & Serbien o. Kosovo: $4,1 \%$ & & & \\
\hline Oberhausen & 211.382 & $25,4 \%$ & Türkei: $28,0 \%$ & 12,3 & & 18.181 \\
\hline & & $14,0 \%$ & Italien: $\quad 6,4 \%$ & & $(\sim 4,4 \%$ & \\
\hline & & & Serbien o. Kosovo: $6,4 \%$ & & der Vereine & 376 \\
\hline & & & Syrien/Arab. Rep.: $\quad 6,3 \%$ & & in $\mathrm{OB}$ ) & \\
\hline & & & $\begin{aligned} \text { Polen: } \quad 5,7 \% \\
\end{aligned}$ & & & \\
\hline Hagen & 188.266 & $\begin{array}{l}37,4 \% \\
16,6 \%\end{array}$ & Türkei: $1,5 \%$ & 12,3 & $\begin{array}{r}75 \\
(\sim 3 \%\end{array}$ & 19.774 \\
\hline & & & Griechenland: $9,4 \%$ & & der Vereine & 343 \\
\hline & & & Rumänien: $8,8 \%$ & & in $\mathrm{HA}$ ) & \\
\hline & & & Syrien/Arab. Rep.: $7,6 \%$ & & & \\
\hline Hamm & 179.571 & $35,6 \%$ & Türkei: $33,9 \%$ & 10,3 & & 18.006 \\
\hline & & $14,1 \%$ & Polen: $13,0 \%$ & & $(\sim 2,7 \%$ & 379 \\
\hline & & & Bulgarien: $7,2 \%$ & & der Vereine & $3 / 9$ \\
\hline & & & $\begin{aligned} \text { Syrien/Arab. Rep.: } & 6,0 \% \\
\text { Rumänien: } & 3,7 \%\end{aligned}$ & & & \\
\hline Gelsen- & 262.528 & $31,2 \%$ & Türkei: $36,1 \%$ & 16,2 & 123 & 16.203 \\
\hline kirchen & & $18,5 \%$ & Polen: $8,3 \%$ & & $(\sim 3,3 \%$ & \\
\hline & & & Rumänien: $7,6 \%$ & & der Vereine & 395 \\
\hline & & & Syrien/Arab. Rep.: 6,7\% & & in GE) & \\
\hline & & & Bunganien. $3,0 \%$ & & & \\
\hline Duisburg & 499.845 & $34,5 \%$ & Türkei: $33,2 \%$ & 14,3 & 145 & 16.881 \\
\hline & & $19,9 \%$ & Bulgarien: $8,2 \%$ & & $(\sim 2,7 \%$ & 393 \\
\hline & & & Syrien/Arab. Rep.: $\quad 6,5 \%$ & & $\begin{array}{r}\text { der vereine } \\
\text { in DU) }\end{array}$ & \\
\hline & & & Polen: $6,2 \%$ & & & \\
\hline
\end{tabular}

Quellen/Datenbasis: Einwohner: MKFFI NRW 2018: 17; Menschen mit MH (= Migrationshintergrund)/Ausländer: MKFFI NRW 2018: 2; Arbeitslosenquote: MKFFI NRW 2018: 8; Anzahl der MSO: Recherchebasis: Vereinsregister 2016; im Jahr 2017 aktualisierte Kontaktdatenliste der religiösen Gemeinden in NRW (Forschungsprojekt „Religiöse Vielfalt in Nordrhein-Westfalen“; Hero et al. 2008); Verfügbares Einkommen pro Einwohner: IT.NRW 2018. 
Förderung von MSO sowie der Existenz von Kommunalen Integrationszentren in allen Kreisen und kreisfreien Städten in NRW, ist jedoch weitestgehend von vergleichbaren kommunalen Rahmenbedingungen auszugehen (II. 3.2).

\subsection{Feldzugänge, Akquise-Strategien, Stichproben und Ausschöpfungsquote}

Das Anliegen dieser Studie ist es, im Rahmen einer Vollerhebung in neun ausgewählten Städten Nordrhein-Westfalens über die Befragung von einzelnen Funktionsträgern bzw. Personen, die die adressierten MSO sehr gut kennen, die Breite der Landschaft der MSO auf der Mesoebene zu untersuchen, um damit zur Schließung einer Forschungslücke beizutragen. Dass MSO bislang wenig erforscht wurden, hängt sicherlich damit zusammen, dass es sich um eine äußerst heterogene Grundgesamtheit von kleinen, in ihrer Existenz bedrohten Vereinen bis hin zu großen, professionell arbeitenden Verbänden handelt, die aufgrund disparater Ressourcenausstattungen sehr unterschiedlich funktionieren und für forschende Personen bekanntermaßen nicht leicht zugänglich sind. Grundsätzlich schwierig ist es, sich über diese Organisationen einen quantitativen Überblick zu verschaffen, denn ihre konkrete Anzahl ist schwer festzustellen (5.1). Zudem ist immer auch zu hinterfragen, ob in Datenbanken oder auf dem Papier registrierte MSO in der Realität auch tatsächlich existieren. So wurde der Autorin der vorliegenden Studie seitens in der Feldphase kontaktierter Ansprechpartner der MSO des Öfteren mitgeteilt, dass die adressierten Migrantenvereine zum Teil „,seit Jahren“ oder ,Jahrzehnten“ nicht mehr bestünden bzw. sich aufgelöst hätten. Gleichwohl waren sie noch im Vereinsregister eingetragen. Auf Nachfrage beim Ministerium der Justiz des Landes Nordrhein-Westfalen wurde der Autorin mitgeteilt, dass Vereinsauflösungen seitens der Registergerichte nicht überprüft würden. Die nach $\S \S 388$ bis 391 FamFG $^{10}$ bei Nicht-Mitteilung der Vereinslöschung vorgesehenen Zwangsgeldverfahren könnten nur dann eingeleitet werden, wenn das Registergericht ,von einem sein Einschreiten rechtfertigenden Sachverhalt glaubhafte Kenntnis erhält, beispielsweise durch Mitteilungen Dritter oder durch

\footnotetext{
${ }^{10}$ Gesetz über das Verfahren in Familiensachen und in den Angelegenheiten der freiwilligen Gerichtsbarkeit.
} 
Zeitungsberichte."11 Vor dem Hintergrund der nicht erfolgenden und grundsätzlich schwierigen Kontrolle ist daher von der Existenz zahlreicher Karteileichen auszugehen.

Im Rahmen der Recherche von MSO und ihrer Kontaktdaten empfiehlt es sich, Post- und E-Mail-Adressen sowie Telefonnummern und nach Möglichkeit Namen von konkreten Ansprechpartnern, wie beispielsweise Vereinsvorsitzenden, ausfindig zu machen. Dieses Vorgehen ermöglicht mehrere Wege der Kontaktaufnahme. Zudem sollte ein solches mit mehreren Akquise-Strategien operierendes Erhebungsdesign mehrsprachig angelegt sein, da Sprachbarrieren in diesem heterogenen Forschungsfeld nicht zu unterschätzen und ernst zu nehmen sind. In andere Sprachen übersetzte Fragebögen wie auch die Durchführung mehrsprachiger Telefoninterviews können die Rücklaufquote deutlich erhöhen bzw. stellen eine Voraussetzung für den Zugang zu allochthonen Zielgruppen und damit den Erfolg von Studien dar (u. a. Simonson et al. 2016b; Priemer et al. 2017; Klöckner 2016; Halm/Sauer 2015; Halm et al. 2012a). Um Nicht-Teilnahmen („UnitNonresponses") aufgrund mangelhafter Deutschkenntnisse zu vermeiden, wurden auch die im Rahmen dieser Studie eingesetzten Fragebögen und Anschreiben auf dem Papier sowie im Online-Umfrageprogramm Unipark in vier weitere Sprachen - Türkisch, Französisch, Englisch und Russisch - übersetzt. Allerdings wurden die anderssprachigen Versionen lediglich von einer überschaubaren Anzahl von Personen genutzt. In rund 88 Prozent der Fälle wurden die Fragen auf Deutsch beantwortet; am zweithäufigsten in Anspruch genommen wurde der türkischsprachige Fragebogen. Der Einsatz der englischen und russischen Varianten erwies sich im Grunde als nicht notwendig (Tab. 5.3).

Tab.5.3 Rücklauf in verschiedenen Sprachen

\begin{tabular}{lcc}
\hline & Häufigkeit & Prozent \\
\hline Deutsch & 226 & 87,9 \\
\hline Türkisch & 21 & 8,2 \\
\hline Französisch & 7 & 2,7 \\
\hline Englisch & 2 & 0,8 \\
\hline Russisch & 1 & 0,4 \\
\hline Gesamt & 257 & 100,0 \\
\hline
\end{tabular}

${ }^{11}$ Aktenzeichen 3824 E - II. 2/18: Antwortschreiben des Ministeriums der Justiz des Landes Nordrhein-Westfalen vom 24.07.2018 auf die seitens der Autorin erfolgte Anfrage per E-Mail, inwieweit Vereinsauflösungen überhaupt kontrolliert werden (können). 
Der Versand der Fragebögen erfolgte mit einem offiziellen Anschreiben auf dem Postweg - zum Teil auch in zwei Sprachen, je nach erkennbarem nationalen Hintergrund einer MSO. Nach 16 Tagen wurde ein Erinnerungsschreiben verschickt. Mit dem Anschreiben verknüpft war das Anliegen, die Adressaten hinsichtlich der mit der Umfrage angestrebten Ermittlung und Veröffentlichung von wichtigen Aktivitäten und speziellem Unterstützungsbedarf - im Sinne eines immateriellen Inzentivs - auf den Nutzen des Projekts für die eigene Organisation hinzuweisen. Hinsichtlich der Bereitschaft den Fragebogen auszufüllen und zurückzusenden, ist es wichtig, den Unterlagen einen bereits adressierten und frankierten Rückumschlag (,Entgelt bezahlt Empfänger") beizulegen, damit den adressierten Personen bzw. Organisationen keine Kosten entstehen.

Eine wesentliche Schwierigkeit bestand darin, die MSO auch tatsächlich zu erreichen. Der Versand der Anschreiben und Fragebögen glich einer ,Trial-andError"-Aktion: Fehlschläge wurden bewusst in Kauf genommen. Bei Befragungen von MSO ist ein zentrales Problem, dass konkrete Ansprechpartner oder Vorstandsvorsitzende sehr häufig nicht ermittelt werden können, was auch auf starke personelle Fluktuationen oder Wechsel der Vereinslokalitäten zurückzuführen sein sollte. Statt einer personalisierten wurde für das Anschreiben daher die unpersönliche Anredeformel „Sehr geehrte Damen und Herren, sehr geehrte Vorstandsvorsitzenden" gewählt und in die Adresszeile unter dem Namen der Organisation „Zu Hd. dem/der Vorsitzenden“ geschrieben.

Zusätzlich erschwerend ist dann, wenn es sich um einen kleinen sogenannten „Wohnzimmerverein“ in einer Privatwohnung handelt, da an der angegebenen Adresse von außen vielfach kein Hinweis auf den Sitz einer MSO vorzufinden ist. In einem solchen Fall weiß der Postzusteller nicht, in welchen Briefkasten er den Umschlag werfen soll: Die Post kommt dann als unzustellbar zurück (,Zurück: Empfänger/Firma unter der angegebenen Anschrift nicht zu ermitteln“). Bei 512 der adressierten Organisationen war die postalische Übersendung der Dokumente erfolglos. Die recherchierten Kontaktdaten der betreffenden MSO wurden aus den Listen gelöscht, wodurch sich die Bruttostichprobe verringerte (Tab. 5.5).

Der Fragebogen sollte von einer mit der Organisation sehr gut vertrauten Person ausgefüllt werden. Diese hatte die Wahl, die Fragen auf dem Papier oder online im Internet unter einem im Anschreiben angegebenen Link auszufüllen. Sowohl die Papier- als auch die Online-Variante wurden in etwa gleich stark von 46 bzw. 41 Prozent der MSO genutzt (Tab. 5.4). Auch erwiesen sich die am Ende der Erhebungsphase zur Erhöhung der Fallzahlen getätigten Versuche, Telefoninterviews durchzuführen, als erfolgreich. Diesbezüglich hilfreich war die Unterstützung einer erfahrenen türkischsprachigen Interviewerin. Als (kontingente) Erfolgsfaktoren entpuppten sich Ausdauer und das richtige Timing, um 
die zumeist freiwillig engagierten, auskunftsfähigen Personen in der Organisation telefonisch $\mathrm{zu}$ erreichen. Während die im Internet recherchierten Telefonnummern scheinbar häufig korrekt waren, traf das auf die E-Mail-Adressen hingegen größtenteils nicht zu. Aus datenschutzrechtlichen Gründen durften die Kommunen die ihnen jeweils zur Verfügung stehenden E-Mail-Verteiler von MSO nicht an die Autorin weiterreichen. Allerdings waren die Kommunalen Integrationszentren in fünf der Erhebungskommunen ${ }^{12}$ bereit bzw. aufgrund vorhandener MSO-Verteiler imstande, per E-Mail zur Beteiligung an der Umfrage aufzurufen und das Anschreiben inklusive den zur Umfrage führenden Link zu kommunizieren. Dass die über die städtischen E-Mail-Verteiler adressierten MSO auch in der Stichprobe vorhanden waren, war insofern gewährleistet, als der Autorin für ihre Recherche Namenslisten der auf kommunaler Ebene bekannten MSO zur Verfügung gestellt wurden oder diese auf den städtischen Internetseiten zu finden waren. $^{13}$

Tab.5.4 Fallzahlen - Anteile der verschiedenen Befragungsmodi

\begin{tabular}{lcc}
\hline & Häufigkeit & Prozent \\
\hline Postalisch & 119 & 46,3 \\
\hline Online & 106 & 41,2 \\
\hline Telefon & 32 & 12,5 \\
\hline Gesamt & 257 & 100,0 \\
\hline
\end{tabular}

Insgesamt sind die Reaktionen auf das Forschungsprojekt positiv zu werten. Selten waren in den MSO tätige Personen verärgert, mit dem Fragebogen konfrontiert zu werden, da die erwünschten Informationen doch auch bei der Stadt zu erfragen oder im Internet zu recherchieren seien. Vereinzelt kritisiert wurde, dass es $\mathrm{zu}$ viele Fragen seien und zuweilen wurde Verwunderung darüber kundgetan, dass der Fragebogen so viele religionsbezogene Aspekte umfasse. Interesse an den Ergebnissen der Studie wurde wiederholt und auch noch nach dem Abschluss der Erhebung bekundet. Vereinzelt wurde der Wunsch einer Kooperation mit der Universität Münster mitgeteilt oder der Unmut darüber, dass die Befragung anonym sei: „Unterstützungsbedarf haben wir! Wie wollen Sie uns helfen, wenn alles anonym ist?"

\footnotetext{
12 Bielefeld, Dortmund, Bochum, Duisburg, Gelsenkirchen.

${ }^{13}$ Doppelte Teilnahmen konnten durch die explizite Ansprache der Vorsitzenden sowie intakte Kommunikationsstrukturen in den Organisationen ausgeschlossen bzw. in sechs Fällen aufgrund von Datenübereinstimmungen identifiziert werden.
} 
Hinsichtlich des Aspektes der Anonymität und des Hinweises einer nicht möglichen Identifizierung von Organisationen und Personen im Anschreiben war auffällig, dass insbesondere die islamischen Verbände DİTIB, IGMG und VIKZ den Freiumschlag für die Rücksendung des ausgefüllten Fragebogens häufig mit einem Stempel versahen, sodass sie ihre Anonymität bewusst aufhoben ${ }^{14}$. Dies könnte als Bedürfnis nach Legitimitätsgewinn und Anerkennung interpretiert werden, da insbesondere die islamischen Verbände in der Vergangenheit und Gegenwart Skepsis und Mistrauen ausgesetzt waren bzw. nach wie vor sind (vgl. II. 4.5).

Eine Nicht-Beteiligung aufgrund von mangelndem Vertrauen wurde in drei Fällen zum Ausdruck gebracht. Diesbezüglich wurde die Studienleiterin (bzw. Autorin dieser Arbeit) einmal auch persönlich telefonisch kontaktiert. In einem anderen Fall wurde der Fragebogen im Freiumschlag unausgefüllt mit einer türkischen Notiz zurückgeschickt, die ins Deutsche übersetzt sinngemäß wie folgt lautete: „Was wir bis heute in guter Absicht getan haben, wurde im Nachhinein immer gegen uns angewendet. Nehmen Sie es uns bitte nicht übel. Es geht um Vertrauen und diesbezüglich haben wir in der Vergangenheit schlechte Erfahrungen gemacht.“

Im Bewusstsein um das Risiko einer geringen Ausschöpfung bemühte sich die Autorin intensiv darum, Funktionsträger in den MSO für eine Teilnahme an der Befragung sowie darüber hinausgehend auch auf kommunaler und Landesebene Fürsprecher zu gewinnen. In Rücksprache mit für die MSO politisch verantwortlichen Personen besuchte die Autorin in den Erhebungskommunen Netzwerktreffen der MSO, Integrationsratssitzungen sowie andere mit und von MSO durchgeführte Veranstaltungen, um dort für die Teilnahme an der Befragung zu werben und ihren Mehrwert hervorzuheben.

Im Großen und Ganzen war die Resonanz auf die Studie seitens der Mitarbeiter in den Kommunalen Integrationszentren positiv. Einige merkten an, dass die MSO sehr häufig befragt würden und daher nicht mit einer hohen Beteiligung zu rechnen sei. Mehrheitlich zeigten sie Interesse an der Studie und den Ergebnissen, da diese auch für die Politik und Fachpraxis relevant seien, was sie auch gegenüber den Organisationen betonten und diese zur Teilnahme anspornten. Derlei positive Haltungen waren insbesondere in den Städten mit intakten Netzwerkund Kooperationsstrukturen, regelmäßig stattfindenden Vernetzungstreffen und vor Ort präsenten Dachverbänden bzw. Zusammenschlüssen von MSO spürbar (Essen, Dortmund, Bielefeld). In zwei Kommunen gestaltete sich der Feldzugang

${ }^{14}$ DİTIB: 6 von insgesamt 10 Fällen im Sample; IGMG: 4 von insgesamt 7 Organisationen; VIKZ: 2 von 2 Fällen. 
schwieriger, da die Stadtpolitik einer aktiven Unterstützung der Umfrage ablehnend gegenüberstand. Begründet wurde dies in einer Kommune damit, dass die im Erhebungsbogen enthaltene Frage nach städtischen Fördermaßnahmen ,ein zu heißes Eisen" sei; eine andere Stadt teilte mit, dass das Kommunale Integrationszentrum nicht über ausreichende personelle Kapazitäten verfüge, was der Autorin aber als fadenscheiniges Argument erschien.

Inwieweit durch die direkte Kontaktaufnahme zu den MSO-Vertretern eine Erhöhung der Beteiligung erwirkt werden konnte, ist aufgrund der Anonymität der Studie nicht eindeutig festzustellen. Der daraus resultierende Erfolg ist aber eher als mäßig einzustufen. Denn im Nachgang der auf den kommunalen Veranstaltungen häufig getätigten Interessenbekundungen und teilweise starken Mitteilungsbedürfnissen erfolgte oftmals keine Rücksendung von ausgefüllten Fragebögen. Dies lässt hinsichtlich der Beantwortung auf die Existenz von wie auch immer gearteten Hemmnissen schließen, wobei natürlich auch Faktoren wie Zeitmangel, Vergessen oder Verlust der Unterlagen nicht auszuschließen sind.

Insgesamt dauerte die Feldphase acht Monate. Diese lange Zeit war ursprünglich nicht vorgesehen. Ende Juli 2018 wurde die Entscheidung getroffen, zur Erhöhung der Fallzahlen die ursprünglich für drei Monate (Mai bis Juli) angesetzte Feldphase auszudehnen und in diesem Zusammenhang zwei weitere Großstädte (Gelsenkirchen und Duisburg) in die Städteauswahl aufzunehmen. Angestrebt war damit nunmehr eine Vollerhebung in neun Kommunen Nordrhein-Westfalens (5.2).

Die Ausgangsstichprobe (Bruttostichprobe) umfasste 1.244 Organisationen, die alle angeschrieben wurden. Davon nichtzustellbar waren 512 Umschläge. Die um stichprobenneutrale Ausfälle aufgrund von Nichtzustellbarkeit zu ermittelnde Nettostichprobe zählte folglich nur noch 732 Organisationen. 257 Organisationsbefragungen wurden realisiert und fungieren als Analysestichprobe. Insgesamt ergibt sich damit eine Ausschöpfungsquote von 32,1 Prozent (Tab. 5.5).

Es zeigt sich, dass in den Städten Bochum, Essen, Dortmund und Bielefeld die höchsten Beteiligungsraten erzielt werden konnten. Auch in sechs anderen als für die Erhebung ausgewählten Kommunen wurde an der Umfrage teilgenommen. Dies ist mit der Weiterleitung der Fragebögen und des Links per E-Mail, aber auch mit der Präsenz von MSO aus anderen Städten auf den Netzwerktreffen zurückzuführen. Deren Teilnahme sollte bei vorhandenem Interesse nicht kategorisch ausgeschlossen werden und mit Blick auf die Erhöhung der Fallzahlen wurde auf den Ausschluss der betreffenden 22 Organisationen verzichtet. Da sie in den für die Erhebungskommunen ermittelten Stichproben nicht vertreten sind, bleiben sie bei der Berechnung der Ausschöpfungsquote unberücksichtigt (Tab. 5.5). 
Tab. 5.5 Stichproben, Rückläufe (pro Kommune), Ausschöpfungsquote (in absoluten und relativen Häufigkeiten)

\begin{tabular}{|c|c|c|c|c|c|c|}
\hline $\begin{array}{l}\text { Erhebungs- } \\
\text { kommunen }\end{array}$ & $\begin{array}{c}\text { Versand } \\
\text { Anschrei- } \\
\text { ben* }^{*} \\
\text { (Brutto- } \\
\text { stichprobe) }\end{array}$ & $\begin{array}{c}\text { Nicht } \\
\text { zustell- } \\
\text { bar }^{* *}\end{array}$ & $\begin{array}{l}\text { Gültige } \\
\text { Fälle } \\
\text { (Netto- } \\
\text { stichprobe) }\end{array}$ & $\begin{array}{c}\text { Rück- } \\
\text { läufer } \\
\text { (absolute } \\
\text { Zahlen) }\end{array}$ & $\begin{array}{c}\text { Rück- } \\
\text { läufer } \\
\text { (pro } \\
\text { Kommune } \\
\text { in \%) }\end{array}$ & $\begin{array}{c}\text { Rücklauf } \\
\text { insgesamt } \\
\text { (in \%) }\end{array}$ \\
\hline Dortmund & 239 & 113 & 126 & 47 & $37,3 \%$ & $20,0 \%$ \\
\hline Essen & 185 & 88 & 97 & 38 & $39,2 \%$ & $16,2 \%$ \\
\hline Bielefeld & 177 & 62 & 115 & 41 & $35,7 \%$ & $17,4 \%$ \\
\hline Bochum & 161 & 83 & 78 & 38 & $48,7 \%$ & $16,2 \%$ \\
\hline Duisburg & 127 & 48 & 79 & 20 & $25,3 \%$ & $8,5 \%$ \\
\hline Gelsenkirchen & 111 & 37 & 74 & 13 & $17,6 \%$ & $5,5 \%$ \\
\hline Oberhausen & 79 & 23 & 56 & 15 & $26,8 \%$ & $6,4 \%$ \\
\hline Hagen & 66 & 29 & 37 & 8 & $21,6 \%$ & $3,4 \%$ \\
\hline Hamm & 99 & 29 & 70 & 15 & $21,4 \%$ & $6,4 \%$ \\
\hline Summe & 1.244 & 512 & 732 & $\underline{235}$ & & $100,0 \%$ \\
\hline $\begin{array}{l}\text { Ausschöp- } \\
\text { fungsquote } \\
\text { (bei Netto- } \\
\text { stichprobe } \\
=732 \text { ) }\end{array}$ & & & & $\underline{32,1 \%}$ & & \\
\hline \multicolumn{7}{|c|}{ Weitere Kommunen } \\
\hline Köln & & & & 16 & & \\
\hline Düsseldorf & & & & 2 & & \\
\hline Bergkamen & & & & 1 & & \\
\hline Bocholt & & & & 1 & & \\
\hline Herne & & & & 1 & & \\
\hline Solingen & & & & 1 & & \\
\hline Summe & & & & 22 & & \\
\hline $\begin{array}{l}\text { Summe insg. } \\
\text { (= Analyse- } \\
\text { stichprobe) }\end{array}$ & & & & 257 & & \\
\hline
\end{tabular}

* Versand Anschreiben: auf Basis recherchierbarer Adressen. Basis: deutsches Vereinsregister 2016; NRWPlural 2017 (Hero et al. 2008 aktualisiert); Internetseiten; kommunale MSO-Listen.

** Nicht zustellbar: Unzustellbarkeit auf dem Postweg (,Zurück: Empfänger/Firma unter der angegebenen Anschrift nicht zu ermitteln").

*** Rückläufer: Inklusive der Teilnahmen an den Online-Befragungen und Telefoninterviews.

Inwieweit es sich bei der Analysestichprobe um eine repräsentative, d. h. „ein verkleinertes, strukturgleiches Abbild der Gesamtmenge“ (Brosius et al. 2016: 61; Herv. weggel.) handelt, ist nicht festzustellen, da über die entsprechenden Merkmale in der Grundgesamtheit keine Aussage getroffen werden kann (ebd.: 61 f.). Grundsätzlich unbekannt ist, was sich hinter den einzelnen 
in den Kontaktlisten aufgeführten MSO für Organisationsstrukturen, Ressourcen, Interessenausrichtungen und Aktivitätsprofile verbergen.

Offenzulegen ist der mit der Wahl des Erhebungsdesigns bzw. der Befragungsmodi (postalisch, telefonisch, online) in Hinblick auf die generierten und ausgewerteten Daten nicht auszuschließende Bias. Denn zum einen ist davon auszugehen, dass in erster Linie etablierte, ressourcenstärkere und (sehr) aktive Organisationen mit recherchierbaren festen Sitzen, Postanschriften und E-MailAdressen erreicht wurden. Zum anderen konnten die angeschriebenen Funktionsträger selbst entscheiden, ob sie an der Umfrage teilnehmen; in diesem Zusammenhang ist davon auszugehen, dass infrastrukturell und bestandsmäBig unterschiedlich aufgestellte Subgruppen der MSO nicht für die Teilnahme gewonnen bzw. motiviert und entsprechend nicht zuverlässig abgebildet werden konnten. In diesem Zusammenhang kann auch eine in der Minderheitensituation auszumachende habituelle Zurückgenommenheit religiöser und migrantischer Organisationen bzw. der in ihnen involvierten Personen relevant sein. Diese mögliche Datenverzerrung ist hinsichtlich der Ergebnisse mitzudenken; sie stellt im Rahmen des ohnehin schwierigen Zugangs zu den MSO für die forschende Einzelperson jedoch ein prinzipielles Dilemma dar.

Die Beteiligung von lediglich 80 religiösen MSO (Tab. 5.6) ist auffällig, insbesondere da sich die Autorin angesichts der leitenden Forschungsfrage dieser Arbeit sehr um ihre Teilnahme bemüht und im Zuge dessen auch Kontakt zu den Zentralen von religiösen Dachorganisationen aufgenommen hat. Hier fielen Resonanzen und Unterstützungsbereitschaft sehr unterschiedlich aus. In Einzelfällen wurde der Studienleiterin mitgeteilt, dass die angesprochenen religiösen Gemeinden grundsätzlich nicht an Befragungen teilnehmen würden. So ist es der Autorin nicht gelungen, die in NRW auch unter den MSO vorhandene religiöse Pluralität durch die Gewinnung von mehr jüdischen, ezidischen, hinduistischen, buddhistischen und christlichen Religionen für die Befragung auszuweiten. Islamische MSO sind in der Substichprobe der religiösen MSO dominierend. Die Überrepräsentation entspricht durchaus auch dem für NRW festzustellenden Gesamtbild (I. 2.1). 
Tab.5.6 Zuordnung der MSO zu religiösen

Traditionen

\begin{tabular}{lcc}
\hline & Häufigkeit & Prozent \\
\hline Islam & 44 & 17,1 \\
\hline Christentum & 17 & 6,6 \\
\hline Alevitentum & 12 & 4,7 \\
\hline Ezidentum & 3 & 1,2 \\
\hline Judentum & 3 & 1,2 \\
\hline Hinduismus & 1 & 0,4 \\
\hline Religiöse MSO & 80 & 31,1 \\
\hline Nicht-religiöse MSO & 177 & 68,9 \\
\hline Gesamt & 257 & 100,0 \\
\hline
\end{tabular}

Open Access Dieses Kapitel wird unter der Creative Commons Namensnennung 4.0 International Lizenz (http://creativecommons.org/licenses/by/4.0/deed.de) veröffentlicht, welche die Nutzung, Vervielfältigung, Bearbeitung, Verbreitung und Wiedergabe in jeglichem Medium und Format erlaubt, sofern Sie den/die ursprünglichen Autor(en) und die Quelle ordnungsgemäß nennen, einen Link zur Creative Commons Lizenz beifügen und angeben, ob Änderungen vorgenommen wurden.

Die in diesem Kapitel enthaltenen Bilder und sonstiges Drittmaterial unterliegen ebenfalls der genannten Creative Commons Lizenz, sofern sich aus der Abbildungslegende nichts anderes ergibt. Sofern das betreffende Material nicht unter der genannten Creative Commons Lizenz steht und die betreffende Handlung nicht nach gesetzlichen Vorschriften erlaubt ist, ist für die oben aufgeführten Weiterverwendungen des Materials die Einwilligung des jeweiligen Rechteinhabers einzuholen.

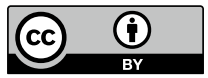

